\title{
Chemical Affinity and the Density of Energy Levels
}

\author{
M. B. Saikhanov \\ Complex Research Institute, Russian Academy of Sciences, Grozny, Chechen Republic, Russia \\ Email: saikhanov musa@mail.ru
}

Received 4 August 2015; accepted 8 September 2015; published 11 September 2015

Copyright @ 2015 by author and Scientific Research Publishing Inc.

This work is licensed under the Creative Commons Attribution International License (CC BY). http://creativecommons.org/licenses/by/4.0/

c) (i) Open Access

\section{Abstract \\ It is shown that the state of chemical equilibrium of a closed system corresponds to the minimum density of its energy levels.}

\section{Keywords}

\section{Chemical Affinity, Closed System, Chemical Equilibrium, Density of Energy Levels, Total Entropy Production, Lyapunov Function}

\section{Introduction}

The concept of chemical affinity $(A)$ was introduced into thermodynamics by Belgian physicist Théophile de Donder in 1922 to represent the driving force of a chemical reaction considered as the irreversible process [1]. Later, it has been found that this concept is very useful for the description of kinetic processes in surface layers, in particular, for investigations of the adsorption and chemisorption processes and the appearance and stable existence of coherent structures in chemical and biological systems [2] [3]. This is indicative of a fundamental character of the concept of chemical affinity as a new intensive thermodynamic parameter determining variation of the number of particles in the system.

The present work is an attempt to understand this important concept on the microscopic level, proceeding from the definition of entropy in quantum statistics (as proposed by Planck in 1925) and the notion of the density of energy levels of a closed system, which plays an important role in modeling the kinetics of nonequilibrium systems [4]-[6].

\section{Main Results and Discussion}

The chemical affinity is defined as the algebraic sum of chemical potentials of the initial reactants and products multiplied by the corresponding stoichiometric coefficients: 


$$
A=-\sum_{\gamma} v_{\gamma} \mu_{\gamma},
$$

where $\mu_{\gamma}$ and $v_{\gamma}$ is the molar chemical potential and stoichiometric coefficient, respectively, of component $\gamma$ participating in a chemical reaction [1]. Taking into account that the stoichiometric coefficients of reactants are negative quantities, while those of products are positive, expression (1) can be rewritten as

$$
A=\sum_{i=1}^{k} v_{i} \mu_{i}-\sum_{j=k+1}^{k+m} v_{j} \mu_{j}
$$

Here $\mu_{i}, \mu_{j}$ and $v_{i}, v_{j}$ are the molar chemical potentials and stoichiometric coefficients of the reactants and the reaction products, respectively. In the state of equilibrium, the chemical affinity is zero; for a reaction proceeding in the forward direction (in excess of reactants), this value is positive, while the reverse reaction (in excess of products) has a negative affinity.

Being an intensive variable (thermodynamic force), the chemical affinity determines an irreversible process related to a change in the composition of a system. In thermodynamics, this variable is considered jointly with the conjugated chemical variable $\xi$ that is called the degree of completion of the reaction and is defined by the following relation [7]:

$$
\mathrm{d} n_{\gamma}=-v_{\gamma} \mathrm{d} \xi,
$$

where $n_{\gamma}$ is the amount (number of moles) of component $\gamma$. Upon dividing both parts of relation (3) by time increment $\mathrm{d} t$, we obtain an expression for the rate of variation of the number of moles $\dot{n}_{\gamma}$ and the reaction rate $\mathrm{v}=\dot{\xi}$ :

$$
\dot{n}_{\gamma}=-v_{\gamma} \dot{\xi}=-v_{\gamma} \mathrm{v} .
$$

Introduction of the chemical affinity and the chemical variable into consideration allows one to reduce the number of variables $n_{\gamma}$ by taking into account the reaction stoichiometry expressed by relations (3) and (4). In particular, for a closed system, the total differential of internal energy $E$ is expressed as

$$
\mathrm{d} E=T \mathrm{~d} S-p \mathrm{~d} V-A \mathrm{~d} \xi,
$$

and the variation of entropy $S$ is expressed as

$$
\mathrm{d} S=\frac{1}{T} \mathrm{~d} E+\frac{p}{T} \mathrm{~d} V+\frac{A}{T} \mathrm{~d} \xi .
$$

Here, the sum of the first and second terms on the right-hand side represents a reversible change in the entropy $\left(d_{e} S\right)$ due to its external flux from outside and the work performed on the system, while the third term is related to the entropy gain $\left(d_{i} S\right)$ inside the system in the presence of a chemical reaction. In order to elucidate the quantum-statistical meaning of the chemical affinity, it is important to consider the irreversible part of the entropy variation

$$
\mathrm{d}_{i} S=(\mathrm{d} S)_{E, V}=\frac{A \mathrm{~d} \xi}{T}
$$

and the corresponding positive definite entropy production [7]:

$$
P=\frac{\mathrm{d}_{\mathrm{i}} S}{\mathrm{~d} t}=\frac{1}{T} A \mathrm{v}>0 .
$$

It turns out that, in the state of chemical equilibrium $(A=0)$, the density $(D)$ of energy levels of a closed system is at minimum, and in the vicinity of equilibrium $D$ is described by a Lyapunov function. The validity of this statement is shown below in several steps.

First, let consider the definition of the density of energy levels of a closed system in quantum statistics [5]. According to this, parameter $D$ is defined as the ratio of the energy interval $\Delta E$, to which the possible energies of elements of the system belong, and the number of these elements represented by their statistical weight $\Delta \Gamma$ :

$$
D(E)=\frac{\Delta E}{\Delta \Gamma}=\Delta E \exp [-S(E)]
$$


where $S(E)$ is the entropy that is the function of energy for states close to the equilibrium [5]. The physical meaning of parameter $D(E)$ is that it characterizes the statistical mean distance (on the energy scale) between the neighboring energy levels of elements of the system. Thus, formula (8) implies that a lower value of parameter $D(E)$ corresponds to a greater number of levels per unit energy interval and, hence, to the energy spectrum with a higher density of states. The density of states in this case is defined as inverse of the density of energy levels, that is, as $D^{-1}=\Delta \Gamma / \Delta E$.

Since the chemical affinity is a linear combination of chemical potentials $\mu_{\gamma}$ (with constant coefficients $v_{\gamma}$ ), it is expedient first to elucidate the quantum-statistical meaning of these potentials. For this purpose, led us take into account relation (3) and rewrite expression (5) in the alternative form as

$$
\mathrm{d} S=\frac{1}{T} \mathrm{~d} E+\frac{p}{T} \mathrm{~d} V-\sum_{\gamma=1}^{k+m} \frac{\mu_{\gamma}}{T} \mathrm{~d} n_{\gamma} .
$$

From Equation (9) we obtain the following expression for the chemical potential:

$$
\mu_{\gamma}=-T\left(\frac{\partial S}{\partial n_{\gamma}}\right)_{E, V, n_{\gamma^{\prime}}}\left(\gamma^{\prime} \neq \gamma\right) .
$$

Using the quantum-statistical definition of entropy on the energy scale of temperature [5],

$$
S=\ln \Delta \Gamma,
$$

and taking into account expression (10), we obtain the following relation:

$$
\mu_{\gamma}=-\frac{T}{\Delta \Gamma}\left(\frac{\partial \Delta \Gamma}{\partial n_{\gamma}}\right)_{E, V, n_{\gamma^{\prime}}}=-T D\left(\frac{\partial D^{-1}}{\partial n_{\gamma}}\right)_{E, V, n_{\gamma^{\prime}}}=T\left(\frac{\partial \ln D}{\partial n_{\gamma}}\right)_{E, V, n_{\gamma^{\prime}}} .
$$

Not that, in deriving relation (11) at the stage of replacement of the statistical weight $\Delta \Gamma$ by parameter $D$ (via relation (8)), we used the energy interval $\Delta E$ that coincides on the order of magnitude with fluctuations in the system energy. In the vicinity of the equilibrium state, this value (as well as the average system energy $E=\bar{E}$ ) can be considered constant [5].

Now let us proceed to calculations of the chemical affinity $(A)$ and total entropy production $(P)$ using the quantum-statistical expression for the chemical potential (11) and formulas (4) and (7). For the chemical affinity, this yields

$$
A=-\sum_{\gamma=1}^{k+m} v_{\gamma} \mu_{\gamma}=-T \sum_{\gamma=1}^{k+m} v_{\gamma}\left(\frac{\partial \ln D}{\partial n_{\gamma}}\right)_{E, V, n_{\gamma^{\prime}}}=\frac{T}{D}\left(\frac{\partial D}{\partial \xi}\right)_{E, V}=T\left(\frac{\partial \ln D}{\partial \xi}\right)_{E, V} .
$$

Note that, in deriving relation (12) at the stage of passing from variables $n_{\gamma}$ to $\xi$,we take into account the dependence of $D$ on variables $n_{\gamma}$ and use relation (3). Then, using relations (7) and (12), we obtain the following expression for the total entropy production:

$$
P=\frac{1}{T} A \dot{\xi}=\left(\frac{\partial \ln D}{\partial \xi}\right)_{E, V} \dot{\xi}=\left(\frac{\mathrm{d} \ln D}{\mathrm{~d} t}\right)_{E, V} .
$$

Since the chemical affinity of a system upon attaining the state of equilibrium is $A=0$, relation (12) with allowance for nonzero absolute temperature $T$ shows that

$$
D=D^{e q}=\text { const . }
$$

\section{Conclusion}

It follows from formula (13) that the positive constant parameter $D$, as expressed by Equation (14), corresponds to zero total entropy production and (in view of its being non-negative) to the absolute minimum $\left(P_{\min }=0\right)$. In addition, it is known from the thermodynamics of nonequilibrium systems that the total entropy production in the vicinity of the equilibrium state is described by a Lyapunov function, $\lambda_{P}=P$ [7] [8]. This implies, in particular, that the system state corresponding to zero total energy production is an attractor. According to expres- 
sion (13), this means that parameter $D$ in this case is a monotonically increasing function of $P$ and, hence, the density of energy levels is also a Lyapunov function $\lambda_{D}=D$, which attains its minimum value in the equilibrium state.

\section{References}

[1] De Donde, Th. and Van Rysselberghe, P. (1936) Thermodynamic Theory of Affinity. Stanford University Press, Stanford University, Stanford.

[2] Defay, R., Prigogine, I. and Bellemans, A. (1966) Surface Tension and Adsorption. Longman, London.

[3] Prigogine, I. (1980) From Being to Becoming: Time and Complexity in the Physical Sciences. Freeman.

[4] Planck, M. (1925) Sitzungsber. Acad. Wiss., Berlin.

[5] Landau, L.D. and Lifshitz, E.M. (1980) Course of Theoretical Physics. Vol. 5, Statistical Physics, Butterworth-Heinemann, Oxford.

[6] Saikhanov, M.B. (2012) International Journal of Modern Physics B, 26, Article ID: 1241005.

[7] Prigogine, I. (1961) Introduction to Thermodynamics of Irreversible Processes. Interscience, New York.

[8] Saikhanov, M.B. (2006) Russian Journal of Physical Chemistry A, 80, 1170-1171. http://dx.doi.org/10.1134/S0036024406070314 